\title{
Stable incidence and continued improvement in short term mortality of Staphylococcus aureus bacteraemia between 1995 and 2008
}

Niels Mejer ${ }^{1,2^{*}}$, Henrik Westh ${ }^{2,3}$, Henrik C Schønheyder ${ }^{4}$, Allan G Jensen ${ }^{5,6}$, Anders R Larsen ${ }^{5}$, Robert Skov ${ }^{5}$, Thomas Benfield ${ }^{1,2,7}$ for the Danish Staphylococcal Bacteraemia Study Group

\begin{abstract}
Background: The objective of this study was to assess temporal changes in incidence and short term mortality of Staphylococcus aureus bacteraemia (SAB) from 1995 through 2008.

Methods: The study was conducted as a nation-wide observational cohort study with matched population controls. The setting was hospitalized patients in Denmark 1995-2008. Uni- and multivariate analyses were used to analyze the hazard of death within 30 days from SAB.

Results: A total of 16330 cases of SAB were identified: 57\% were hospital-associated (HA), 31\% were community-acquired (CA) and 13\% were of undetermined acquisition. The overall adjusted incidence rate remained stable at 23 per 100000 population but the proportion of SAB cases older than 75 years increased significantly. Comorbidity in the cohort as measured by Charlson comorbidity index (CCl) score and alcohol-related diagnoses increased over the study period. In contrast, among the population controls the CCl remained stable and alcohol-related diagnoses increased slightly. For HA SAB crude 30-day mortality decreased from $27.8 \%$ to $21.8 \%$ (22\% reduction) whereas the change for CA SAB was small (26.5\% to $25.8 \%$ ). By multivariate Cox regression, age, female sex, time period, $\mathrm{CCl}$ score and alcohol-related diagnoses were associated with increased mortality regardless of mode of acquisition.
\end{abstract}

Conclusions: Throughout a 14-year period the overall incidence of SAB remained stable while the overall short term prognosis continued to improve despite increased age and accumulation of comorbidity in the cohort. However, age and comorbidity were strong prognostic indicators for short term mortality.

Keywords: Bacteraemia, Epidemiology, Incidence, Mortality, Comorbidity, Alcoholism, Staphylococcus aureus, Charlson comorbidity index

\section{Background}

During the last decades general improvements in health and socioeconomic conditions in many industrialized countries have led to an older population [1]. In Denmark, individuals older than 65 years increased only modestly from $16 \%$ to $18 \%$ between 1990 and 2010 but the proportion of individuals older than 90

\footnotetext{
* Correspondence: nmejer@dadlnet.dk

'Department of Infectious Diseases, Hvidovre University Hospital, Hvidovre, Denmark

${ }^{2}$ Faculty of Health Sciences, University of Copenhagen, Copenhagen,

Denmark

Full list of author information is available at the end of the article
}

increased by $72 \%$ [2]. Age is one of the strongest determinants of risk of infectious disease [3]. Older age is further associated with an increased risk and prevalence of comorbidity [4]. The combination of an ageing population and associated comorbidity may lead to an increase in the incidence of sepsis and bloodstream infection. This was evident throughout the 1980's and 1990's for Staphylococcus aureus bacteraemia $(\mathrm{SAB})$ and bacteraemia in general as shown by us and others [5-7]. During the same time frame, the prevalence of several other risk factors has increased. These include increased use of cancer chemotherapy, immunosuppressive therapy, intravascular devices, and invasive medical

\section{Biomed Central}


and surgical procedures. Taken together ageing, accumulation of comorbidity and other risk factors may influence both the incidence and prognosis of SAB.

Although much attention has been drawn towards methicillin-resistant $S$. aureus (MRSA) in recent years, methicillin-susceptible $S$. aureus (MSSA) continues to constitute the most common type of $\mathrm{SAB}$ in most parts of the world. In Denmark MRSA SAB is infrequent. Using a nationwide cohort we investigated contemporary changes in the incidence rate (IR) of SAB (> 99\% MSSA), temporal changes in age and underlying morbidity and assessed the effect of each on short term outcome from MSSA SAB from 1995 to 2008.

\section{Methods}

\section{Study population}

The register-based matched cohort was created by merging data from four national registries based on the unique civil registration number assigned to each Danish citizen [8]. The study was approved by the Ethics Committee for the Municipalities of Copenhagen and Frederiksberg (01-369 / 93) and the Danish Data Protection Agency (2009-41-4179).

The Danish Staphylococcal Bacteraemia Study Group has carried out a continuous, nationwide registration of SAB in Denmark since 1956 [6,9]. The present study defined a case as an individual with a first episode of SAB identified via the registry between January 1, 1995 and December 31, 2008. Discharge records for each admission with $\mathrm{SAB}$ were reviewed [6,9]. We defined hospitalassociated SAB (HA SAB) as an infection acquired after 48 hours of hospitalisation, an infection in a patient in ambulatory care in a specialized hospital department, or a case residing in a nursing home. Community-acquired $\mathrm{SAB}(\mathrm{CA} \mathrm{SAB})$ was defined as an infection present at the time of hospitalization in patients not meeting the definition of HA SAB. Methicillin resistance was determined by antimicrobial susceptibility testing and confirmed by the presence of the mecA gene [10,11].

Information on the number of persons alive in Denmark on January 1 from 1995 through 2008 was obtained from Statistics Denmark (Danmarks Statistik at http:// www.dst.dk). These included total number and subgroups defined by age and gender.

The Danish Civil Registration System which is updated daily, tracks changes in vital status and migration for the entire Danish population [8]. For each case, we randomly selected 10 control subjects, matched by age (year of birth) and sex. We used the risk set sampling technique (i.e. eligible control subjects had to be alive and at risk of a first hospitalization with $\mathrm{SAB}$ on the date the corresponding case was admitted [12]).

Data on comorbidity was collected from the National Patient Registry. This registry is updated monthly and holds information on all hospital admissions and discharge diagnoses of all patients treated in a Danish hospital since January 1, 1977 as well as all outpatient contacts since January 1, 1995. The International Classification of Diseases (ICD)-8 was used from 1977 through 1993; and ICD-10 from 1994. Comorbidity prior to the $\mathrm{SAB}$ admission was assessed by estimating the Charlson comorbidity index (CCI) score [13,14]. Briefly, the CCI is a validated score system used to asses patients' comorbid conditions in longitudinal studies [15], and takes into account both the number and severity of comorbid disease. We calculated the CCI score for cases and controls by gathering diagnoses from the National Patient Registry from up to 10 years prior to the $\mathrm{SAB}$ index date and excluding diagnoses from the admission with SAB. To avoid the influence of re-registration of diagnoses, each of the 17 Charlson diagnostic categories could only contribute one time to the overall index. We defined three levels of comorbidity for categorizing patients in this study: none (patients without underlying disease), intermediate (weighted index of comorbidity CCI 1-2) and high $(\mathrm{CCI} \geq 3)$. Alcohol related comorbidity is not included in the CCI score and was assessed separately as previously described $[16,17]$. We considered the following diagnosis codes: for ICD-10 classification: F10, G31.2, G62.1, G72.1, I42.6, K29.2, K86.0, K70, R78.0, T51, Z72.1, and for ICD-8 classification: 291 (Alcoholic psychosis), 303 (Alcoholism), 979 (Alcohol in combination with specified medicinal agents), 980 (Toxic effect of alcohol), 577.10 (Chronic pancreatitis due to alcohol), 571.09 (Cirrhosis of the liver due to alcohol), 571.10 (Steatosis of the liver due to alcohol). ICD-10 K70 (cirrhosis) is included in the CCI score but was only assessed for alcohol-related conditions.

\section{Statistics}

Annual data were divided into five periods (1995-1997, 1998-2000, 2001-2003, 2004-2006 and 2007-2008) for the assessment of temporal changes, and age was divided into 6 groups $(<1,1-15,16-35,36-55,56-75,>75)$. Using the cumulative incidence proportion method to calculate incidence rates, that assumes that all individuals of a given birth cohort remain alive for a given year, indicated that incidence rates increased over time. However, annual survival rates for individuals older than 65 improved significantly during the study, leading to an average increase in days at risk of SAB in the older age strata in more recent time periods. E.g. among population controls $>75$ years the 30 -day- and 1-year mortality proportion decreased from $7,0 \%$ and $51,4 \%$ in $1995-$ 1997 to $1,2 \%$ and $12,8 \%$ in 2007-2008, respectively. Therefore, we adjusted the number of days at risk in each gender- and age stratum by a correction factor derived from annual survival among the population 
controls. Incidence rates with $95 \%$ CIs were calculated based on these adjusted data. Categorical variables were compared by chi-square test. Ninety days survival curves were constructed by the Kaplan-Meier method, and the proportional hazards assumption was evaluated visually on survival plots. Mortality rate ratios with 95\% CIs were calculated using Cox proportional hazards models. Unvariate and multivariate Cox regression was performed to determine the mortality rate ratios within 30 days of SAB. All variables in the multivariate analysis were analysed using the forced entry procedure. Analyses were performed using PASW v.18 (SPSS Inc., Chicago, IL, USA).

\section{Results}

During the study period, 17450 individuals were hospitalized with a first episode of SAB. A total of 820 of these were excluded due to a temporary or invalid personal identifier $(\mathrm{n}=652)$, negative follow up time $(n=142)$, no sample date $(n=18)$ or a duplicated person identifier $(n=8)$. MRSA was rare $(<1 \%$, Table 1$)$.

\section{Characteristics of cases and controls}

Fifty-seven percent of cases were HA and $31 \%$ were CA. In $13 \%$ of cases the place of acquisition could not be determined. The characteristics of HA and CA cases are shown in Table 1. Overall, patients were in their midsixties and the majority were male.

The proportion of cases older than 75 years increased from $23.2 \%$ in $1995-1997$ to $29.8 \%$ in $2007-2008$ (p = $0.0001)$, and the proportion of cases aged 1-55 years decreased during the same period from $37.1 \%$ to $28.1 \%$ $(\mathrm{p}=0.0001)$. The proportion of cases aged $<1$ years and 56-75 years were stable.

The proportion of cases who had a CCI score >0 increased during the study period from $60.8 \%$ in $1995-$ 1997 to $69.5 \%$ in $2007-2008$ ( $\mathrm{p}=0.0001$ ), of which the proportion of individuals with a CCI score $>2$ increased from $19 \%$ to $32 \%$ ( $\mathrm{p}=0.0001)$. The proportion of cases with a CCI score increased with age although fewer cases older than 75 years compared to the 56-75 year old stratum had a CCI score greater than $0(72.0 \%$ vs. $74.8 \%$, $\mathrm{p}=0.001)$. Patients with HA SAB had higher CCI scores than CA SAB. Among HA SAB 70,0\% had a CCI greater than 0 compared to $54,8 \%$ among CA SAB ( $\mathrm{p}=0.0001$ ).

The proportion of cases with pre-admission alcoholrelated diagnoses increased from $7 \%$ to $12 \%$ between 1995-1997 and 2007-2008 ( $\mathrm{p}=0.0001)$. Alcohol-related diagnoses were more frequent among CA SAB compared to HA SAB ( $\mathrm{p}=0.0001)$.

The distribution of CCI ( $\mathrm{p}=0.0001)$ and alcohol-related disorders $(\mathrm{p}=0.0001)$ among the age- and sex matched controls differed from cases. For controls $23 \%$ had a CCI score of $1-2$ and only $6.0 \%$ had a CCI score $>2$. Over time
Table 1 Characteristics of cases with hospital-associated and community-acquired Staphylococcus aureus bacteraemia in Denmark from 1995-2008

\begin{tabular}{|c|c|c|}
\hline & $\begin{array}{c}\text { Hospital-associated } \\
\text { SAB N = } 9412(56.6 \%)\end{array}$ & $\begin{array}{l}\text { Community-acquired } \\
\text { SAB N = 5074 (30.5\%) }\end{array}$ \\
\hline \multicolumn{3}{|l|}{ MRSA } \\
\hline No & $9332(99.2 \%)$ & 5039(99.3\%) \\
\hline Yes & $80(0.8 \%)$ & $35(0.7 \%)$ \\
\hline \multicolumn{3}{|c|}{ Age, years } \\
\hline$<1$ & $337(3.6 \%)$ & $58(1.1 \%)$ \\
\hline $1-15$ & $232(2.5 \%)$ & $235(4.6 \%)$ \\
\hline $16-35$ & $535(5.7 \%)$ & 405(8.0\%) \\
\hline $36-55$ & 1733(18.4\%) & $938(18.5 \%)$ \\
\hline $56-75$ & $3982(42.3 \%)$ & 1734(34.2\%) \\
\hline$>75$ & $2593(27.5 \%)$ & 1704(33.6\%) \\
\hline \multicolumn{3}{|l|}{ Sex } \\
\hline Female & 3755(39.9\%) & 1927(38.0\%) \\
\hline Male & $5657(60.1 \%)$ & $3147(62.0 \%)$ \\
\hline \multicolumn{3}{|l|}{$\mathrm{CCl}$ score } \\
\hline 0 & $2822(30.0 \%)$ & $2293(45.2 \%)$ \\
\hline $1-2$ & $3868(41.1 \%)$ & $1801(35.5 \%)$ \\
\hline$>2$ & $2722(28.9 \%)$ & $980(19.3 \%)$ \\
\hline
\end{tabular}

\section{Alcohol-related}

disorders

\begin{tabular}{lcc} 
No & $8585(91.2 \%)$ & $4532(89.3 \%)$ \\
Yes & $827(8.8 \%)$ & $542(10.7 \%)$ \\
\hline Year & $1893(20.1 \%)$ & $989(19.5 \%)$ \\
$1995-1997$ & $1992(21.2 \%)$ & $1261(24.9 \%)$ \\
$1998-2000$ & $2010(21.4 \%)$ & $893(17.6 \%)$ \\
$2001-2003$ & $2171(23.1 \%)$ & $1155(22.8 \%)$ \\
$2004-2006$ & $1346(14.3 \%)$ & $776(15.3 \%)$ \\
$2007-2008$ & &
\end{tabular}

SAB: Staphylococcus aureus bacteraemia; MRSA: Methicillin-resistant Staphylococcus aureus.

$\mathrm{CCl}$ : Charlson comorbidity index.

the proportion of controls with a CCI score $>0$ decreased from $31.4 \%$ in $1995-1997$ to $28.0 \%$ in $2007-2008$ ( $\mathrm{p}=$ 0.0001 ), while the proportion of controls with preadmission alcohol-related diagnoses increased from 1.5\% to $2.1 \%(\mathrm{p}=0.0001)$.

\section{Incidence rates of SAB}

The adjusted overall IR (22.7/100 000 population (95\% CI: 22.4-23.1/100 000)) of first time SAB remained constant from 1995 to 2008 (Table 2). IRs increased with age and were higher among males (27.9/100.000 population (95\%CI: 27.4-28.5/100 000)) than females (17.6/ 100.000 population (95\%CI: $17.2-18.1 / 100.000)$ ). The IRs decreased slightly from 1995 to 2008 among persons 
Table 2 Incidence rates* of Staphylococcus aureus bacteraemia in Denmark from 1995-2008

\begin{tabular}{|c|c|c|c|c|c|}
\hline Year & 1995-1997 & $1998-2000$ & 2001-2003 & 2004-2006 & 2007-2008 \\
\hline All & $23.4(22.6-24.2)$ & 23.5(22.8-24.3) & $21.2(20.5-21.9)$ & $23.4(22.6-24.1)$ & 21.8(20.9-22.7) \\
\hline \multicolumn{6}{|l|}{ Sex } \\
\hline Male & $28.3(27.1-29.5)$ & $29.4(28.2-30.6)$ & $26.1(25.0-27.2)$ & $28.6(27.5-29.8)$ & $27.1(25.8-28.6)$ \\
\hline Female & 18.7(17.8-19.7) & 17.9(17.0-18.8) & 16.5(15.6-17.4) & 18.2(17.3-19.1) & 16.6(15.5-17.7) \\
\hline \multicolumn{6}{|l|}{ Age, years } \\
\hline$<1$ & $40.5(31.8-49.2)$ & $37.1(28.7-45.6)$ & $42.3(33.2-51.4)$ & $57.0(46.3-67.6)$ & $52.6(40.1-65.1)$ \\
\hline $1-15$ & $5.3(4.4-6.1)$ & $4.6(3.8-5.4)$ & $3.1(2.5-3.7)$ & $3.4(2.8-4.1)$ & $3.5(2.7-4.4)$ \\
\hline $16-35$ & $7.3(6.5-8.1)$ & $6.9(6.1-7.6)$ & $4.3(3.6-4.9)$ & $4.3(3.7-4.9)$ & $4.0(3.2-4.7)$ \\
\hline $36-55$ & 16.8(15.6-18.1) & $15.7(14.5-16.8)$ & 13.4(12.3-14.4) & 13.7(12.6-14.8) & $13.5(12.2-14.8)$ \\
\hline $56-75$ & $58.9(55.8-62.0)$ & 51.6(48.9-54.3) & $45.3(42.9-47.7)$ & $46.7(44.3-49.0)$ & $43.5(40.8-46.2)$ \\
\hline$>75$ & $114.2(106.3-122.0)$ & $120.7(113.4-128.1)$ & $112.7(105.9-119.5)$ & $126.7(119.6-133.8)$ & 107.7(99.7-115.6) \\
\hline
\end{tabular}

* Adjusted incidence rates per 100000 population years, (): 95\% Confidence interval.

aged 1-75 years and were stable among cases aged $<1$ and $>75$ years (Table 2 ).

\section{Mortality}

The overall cumulative mortality among cases within 30 days from $\mathrm{SAB}$ was $25.7 \%$. The median survival of $\mathrm{HA}$, $\mathrm{CA}$ and undetermined acquisition SAB was 556 days (501-610), 836 days (721-951) and 476 days (386-566), respectively.

There was a significant impact of origin, age, gender, CCI, alcohol-related disorders and time period on mortality (Tables 3 and 4). Due to few deaths among SAB patients $<36$ years the periodic 30 day mortality proportions in these age groups were not presented. The highest impact on mortality was noted with age (Figure 1). The impact diminished in multivariate analyses, but remained the most influential. Second and third highest impact was seen with alcohol-related disorders and comorbidity assessed by CCI (Figure 2). By multivariate analyses the impact of alcohol-related disorders increased after adjustment for age whereas the impact of CCI decreased. This was seen among both HA and CA SAB. Multivariate analyses showed improved survival over time particularly among cases of HA but also CA bacteraemia. Gender had the lowest impact on mortality and men had lower mortality in the adjusted analysis.

\section{Discussion}

In a cohort of more than 16000 individual episodes of $\mathrm{SAB}$, we show that the incidence of first time $\mathrm{SAB}$ remained stable across age groups, origin and degree of comorbidity from 1995 to 2008 after several decades of increasing incidence rates. Further, we document that overall short term survival rates continued to improve although mortality rates were higher with increasing age and level of comorbidity.

Table 3 Thirty-day mortality proportions from Staphylococcus aureus bacteraemia in Denmark between 1995 and 2008

\begin{tabular}{|c|c|c|c|c|c|}
\hline Year & 1995-1997 & $1998-2000$ & 2001-2003 & 2004-2006 & 2007-2008 \\
\hline All & $0.27(0.25-0.28)$ & $0.26(0.25-0.28)$ & $0.26(0.24-0.27)$ & $0.26(0.25-0.27)$ & $0.23(0.21-0.25)$ \\
\hline \multicolumn{6}{|l|}{ Sex } \\
\hline Female & $0.29(0.26-0.31)$ & $0.27(0.25-0.30)$ & $0.28(0.26-0.30)$ & $0.28(0.25-0.30)$ & $0.25(0.22-0.28)$ \\
\hline Male & $0.26(0.24-0.28)$ & $0.25(0.24-0.27)$ & $0.24(0.22-0.26)$ & $0.25(0.23-0.27)$ & $0.22(0.20-0.24)$ \\
\hline \multicolumn{6}{|l|}{ Age, years } \\
\hline $36-55$ & $0.19(0.17-0.22)$ & $0.17(0.14-0.19)$ & $0.15(0.12-0.18)$ & $0.15(0.12-0.17)$ & $0.11(0.08-0.14)$ \\
\hline $56-75$ & $0.29(0.27-0.32)$ & $0.26(0.24-0.28)$ & $0.23(0.21-0.25)$ & $0.24(0.22-0.26)$ & $0.23(0.21-0.26)$ \\
\hline$>75$ & $0.45(0.42-0.48)$ & $0.44(0.41-0.47)$ & $0.42(0.39-0.45)$ & $0.42(0.39-0.45)$ & $0.37(0.33-0.40)$ \\
\hline \multicolumn{6}{|l|}{$\mathrm{CCl}$} \\
\hline 0 & $0.20(0.18-0.22)$ & $0.20(0.18-0.23)$ & $0.18(0.15-0.20)$ & $0.16(0.14-0.18)$ & $0.17(0.15-0.20)$ \\
\hline $1-2$ & $0.30(0.28-0.32)$ & $0.29(0.27-0.31)$ & $0.29(0.26-0.31)$ & $0.27(0.25-0.30)$ & $0.25(0.22-0.28)$ \\
\hline$>2$ & $0.34(0.31-0.38)$ & $0.30(0.27-0.33)$ & $0.30(0.27-0.33)$ & $0.34(0.31-0.36)$ & $0.26(0.23-0.29)$ \\
\hline Alcohol-related disorders & $0.34(0.28-0.40)$ & $0.32(0.27-0.37)$ & $0.31(0.26-0.35)$ & $0.32(0.27-0.37)$ & $0.30(0.24-0.35)$ \\
\hline
\end{tabular}

CCl: Charlson comorbidity index, (): 95\% Confidence interval. 
Table 4 Multivariate analysis of factors associated with mortality among cases of hospital-associated and communityacquired Staphylococcus aureus bacteraemia in Denmark from 1995-2008

\begin{tabular}{|c|c|c|c|c|}
\hline & \multicolumn{2}{|c|}{ Hospital-associated SAB } & \multicolumn{2}{|c|}{ Community-acquired SAB } \\
\hline & 30-d mortality (\%) & Adjusted MRR $(95 \% \mathrm{Cl})$ & 30-d mortality (\%) & Adjusted MRR $(95 \% \mathrm{Cl})$ \\
\hline Overall & $2368 / 9412(25.2 \%)$ & & $1439 / 5074(28.4 \%)$ & \\
\hline \multicolumn{5}{|l|}{ Age, years } \\
\hline $1>$ & 19/337 (5.6\%) & $0.45(0.28-0.73)$ & $1 / 58(1.7 \%)$ & $0.13(0.02-0.93)$ \\
\hline $1-15$ & 9/232 (3.9\%) & $0.27(0.14-0.53)$ & $1 / 235(0.4 \%)$ & $0.03(0.04-0.23)$ \\
\hline $16-35$ & $32 / 535(6.0 \%)$ & $0.39(0.27-0.56)$ & 15/405 (3.7\%) & $0.25(0.14-0.42)$ \\
\hline $36-55$ & 282/1733 (16.3\%) & 1.0 & $152 / 938(16.2 \%)$ & 1.0 \\
\hline $56-75$ & 983/3982 (24.7\%) & $1.7(1.5-1.9)$ & 483/1734 (27.9\%) & $1.8(1.5-2.2)$ \\
\hline$>75$ & 1043/2593 (40.2\%) & $3.2(2.8-3.7)$ & 787/1704 (46.2\%) & $3.6(3.0-4.4)$ \\
\hline \multicolumn{5}{|l|}{ Sex } \\
\hline Female & 988/3755 (26.3\%) & 1.0 & 616/1927 (32.0\%) & 1.0 \\
\hline Male & 1380/5657 (24.4\%) & $0.90(0.83-0.98)$ & $823 / 3147(26.2 \%)$ & $0.82(0.74-0.92)$ \\
\hline \multicolumn{5}{|l|}{$\mathrm{CCl}$ score } \\
\hline 0 & $527 / 2822(18.7 \%)$ & 1.0 & 435/2293 (19.0\%) & 1.0 \\
\hline $1-2$ & 1049/3868 (27.1\%) & $1.3(1.1-1.4)$ & 606/1801 (33.6\%) & $1.3(1.1-1.5)$ \\
\hline$>2$ & 792/2722 (29.1\%) & $1.4(1.2-1.6)$ & $398 / 980(40.6 \%)$ & $1.6(1.4-1.9)$ \\
\hline \multicolumn{5}{|c|}{ Alcohol-related disorders } \\
\hline No & $2113 / 8585$ (24.6\%) & 1.0 & $1251 / 4532(27.6 \%)$ & 1.0 \\
\hline Yes & 255/827 (30.8\%) & $1.7(1.5-2.0)$ & 188/542 (34.7\%) & $1.9(1.6-2.2)$ \\
\hline \multicolumn{5}{|l|}{ Year } \\
\hline 1995-1997 & $526 / 1893(27.8 \%)$ & 1.0 & $262 / 989(26.5 \%)$ & 1.0 \\
\hline 1998-2000 & 502/1992 (25.2\%) & $0.83(0.74-0.94)$ & $365 / 1261$ (28.9\%) & $1.00(0.85-1.17)$ \\
\hline $2001-2003$ & $512 / 2010(25.5 \%)$ & $0.79(0.70-0.89)$ & $257 / 893(28.8 \%)$ & $0.89(0.75-1.06)$ \\
\hline $2004-2006$ & $535 / 2171(24.6 \%)$ & $0.74(0.66-0.84)$ & $355 / 1155$ (30.7\%) & $0.90(0.76-1.05)$ \\
\hline 2007-2008 & 293/1346 (21.8\%) & $0.65(0.56-0.75)$ & $200 / 776(25.8 \%)$ & $0.74(0.61-0.89)$ \\
\hline
\end{tabular}

SAB: Staphylococcus aureus bacteraemia; MRR: Mortality rate ratio; Cl: confidence interval, CCl: Charlson comorbidity index.

The stable incidence of SAB in the observation period was also found elsewhere [18], but is somewhat surprising. A continued increase in incidence was expected given an increasing larger proportion of elderly with more comorbidity and in a time with more chemotherapy, immunosuppressive treatment and increased use of invasive procedures and devices. A possible explanation may be that although the proportion of elderly is expanding, the elderly are ageing healthier than just a decade ago. Our analysis of the control population certainly showed a considerable improvement in survival over time in support of this.

This study identified high risk of SAB among persons $<1$ year. This finding is consistent with other contemporary studies [19-21] and warrants further studies on $\mathrm{SAB}$ among neonates.

The short term mortality associated with SAB decreased by $14 \%$ from $1995-1997$ to $2007-2008$ in spite of the presence of more comorbidity and increased age. In fact, the total number of deaths attributed to
SAB declined for the first time since 2000 because the incidence rate also remained stable. We speculate that the improved gains may have been accomplished through healthier ageing and improved treatment of comorbid diseases, but many factors may be at play. Still, further improvement in the treatment of SAB must be accomplished if the improvement in the prognoses of $\mathrm{SAB}$ is to follow the improvement seen in the agematched general population.

The strengths of this study lies in its size, the longitudinal and population-based design, the low frequency of MRSA which enabled a clean focus on MSSA, the uniform registration system, and the inclusion of randomly selected age- and sex matched population controls that allowed for adjustment of a baseline mortality risk. There are also potential limitations. First, the discharge data, which the assessment of comorbidity was built upon, may have contained errors, but this misclassification bias would be non-differential between cases and controls. Second, the temporal rise in CCI and alcohol- 


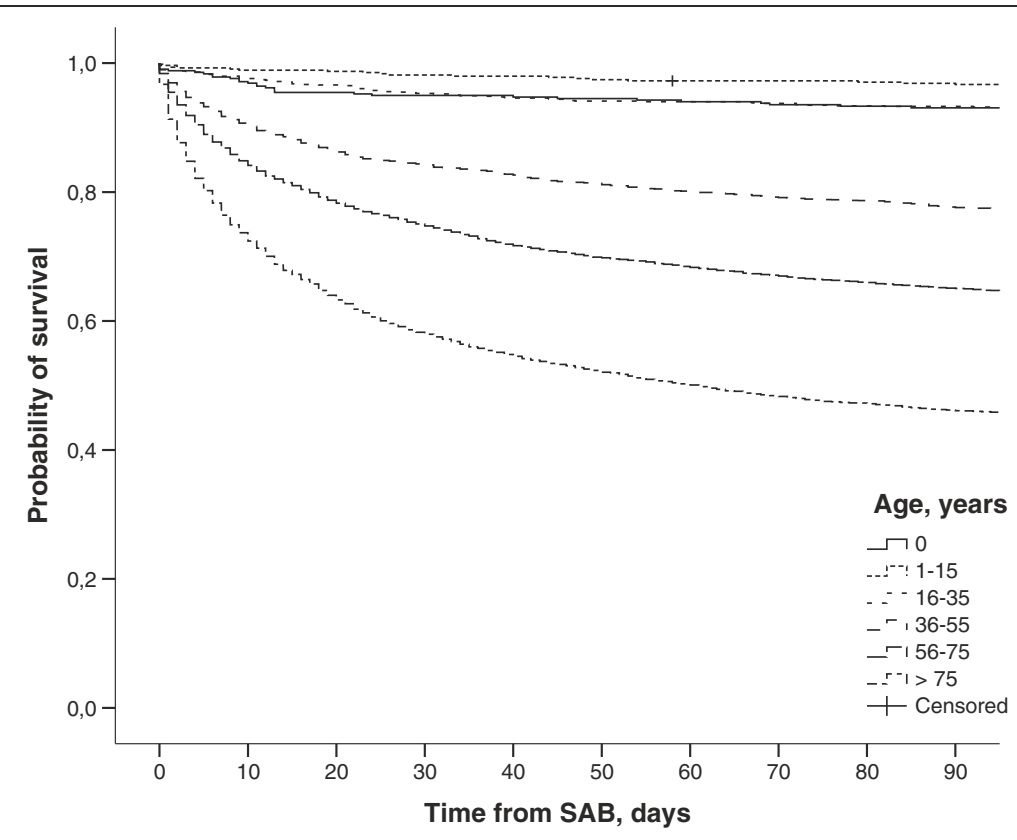

Figure 1 Survival curves of 16630 cases with Staphylococcus aureus bacteraemia between 1995 and 2008 according to age.

related diagnoses among cases could have been caused by an intensified registration of diagnoses. This is, however, unlikely since diagnoses included in the CCI score among population controls remained constant. Third, using CCI as a aggregated estimate of comorbidity has limitations: the CCI unlikely included all comorbid diseases and the improved prognosis of many diseases including cancer and HIV/AIDS in the study period may have overadjusted the impact of CCI on mortality late in the observation period compared to early. Fourth, surveillance bias among the increasing proportion of cases with pre-SAB comorbidity could explain part of the improved prognosis of $\mathrm{SAB}$.

Fifth, a potential major limitation of the study, is its classification of HA and CA SAB. The present study included community onset healthcare-related $S A B$ as

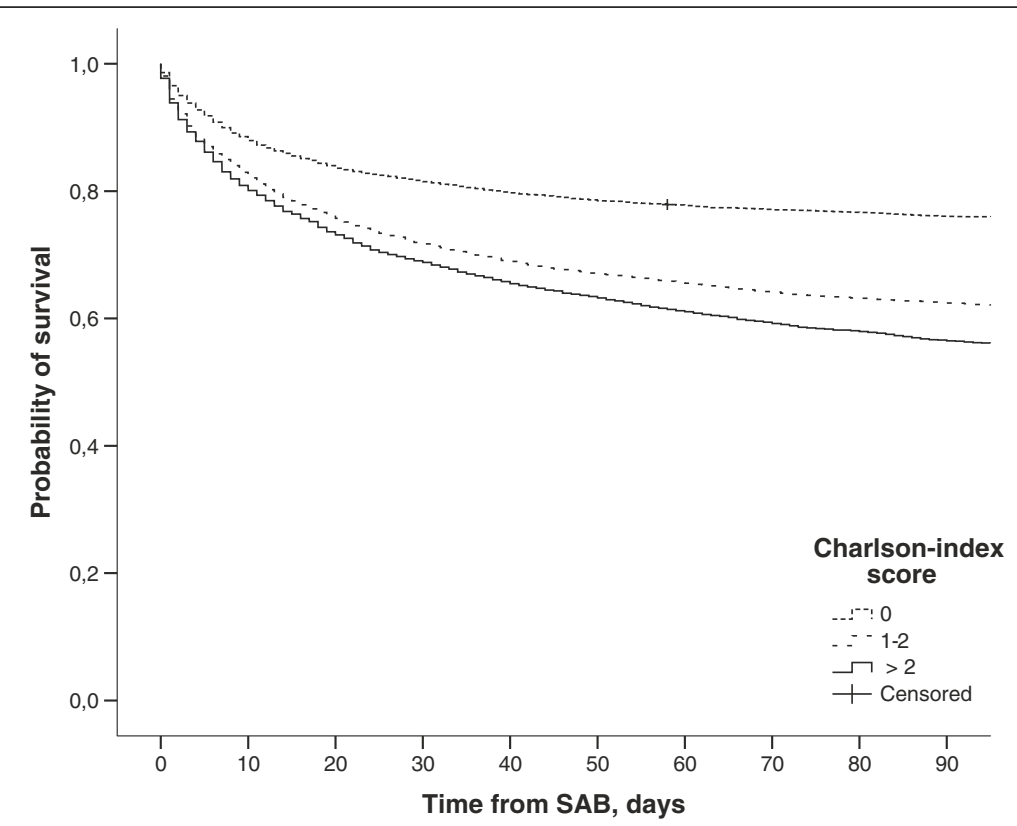

Figure 2 Survival curves of 16630 cases with Staphylococcus aureus bacteraemia between 1995 and 2008 according to Charlson comorbidity index score. 
HA SAB which could make comparison to data from the recent literature difficult.

\section{Conclusion}

In conclusion, the overall IR of first time SAB did not increase from 1995 to 2008 as would be expected from the changing epidemiological profile of the Danish population and the increased medical activity. Further, the short term prognosis of SAB improved; especially among HA SAB. Although the over-all risk of dying from $S A B$ declined, the short term mortality of $S A B$ is still high, especially among the old and comorbid, which continues to constitute a growing proportion of the $\mathrm{Da}$ nish SAB patients. This study underscores the importance of adjustment for age and comorbidity when comparing risk of and mortality from SAB sequentially or between countries. In addition, it highlights the potential benefits of future studies in prevention and treatment of SAB among the old and comorbid patients.

\section{Abbreviations}

SAB: Staphylococcus aureus bacteraemia; MRSA: Methicillin-resistant Staphylococcus aureus; MSSA: Methicillin-susceptible Staphylococcus aureus; CCl: Charlson comorbidity index; HA: Hospital-associated; CA: Communityacquired; IR: Incidence rate; Cl: Confidence interval.

\section{Competing interests}

A.G. Jensen has been working at Pfizer Denmark since 2005 but Pfizer Denmark has no financial interests in- or support to the present study. All other authors declare that they have no competing interests.

\section{Authors' contributions}

TB conceived the study idea. NM and TB designed the study. NM, HW, HCS, $A G J, A R L, R S$ and TB collected the data and NM analyzed the data. NM and TB interpreted the findings. NM wrote the initial draft. All authors edited the manuscript and approved the final version. TB is the guarantor.

\section{Acknowledgments}

The authors thank the staff at the participating clinical microbiological departments for their contribution, continuous support and enthusiasm

\section{Author details}

'Department of Infectious Diseases, Hvidovre University Hospital, Hvidovre, Denmark. ${ }^{2}$ Faculty of Health Sciences, University of Copenhagen, Copenhagen, Denmark. ${ }^{3}$ Department of Clinical Microbiology, Hvidovre University Hospital, Hvidovre, Denmark. ${ }^{4}$ Department of Clinical Microbiology, Aarhus University Hospital, Aalborg, Denmark. ${ }^{5}$ Staphylococcal Laboratory, Statens Serum Institut, Copenhagen, Denmark. ${ }^{6}$ Pfizer, Ballerup, Denmark. ${ }^{7}$ Clinical Research Centre, Hvidovre University Hospital, Hvidovre, Denmark.

Received: 4 March 2012 Accepted: 11 October 2012 Published: 17 October 2012

\section{Reference}

1. Dini E, Goldring S: Estimating the changing population of the 'oldest old'. Popul Trends 2008, 132:8-16.

2. Anonymous: Population figures. Danmarks Statistik 2011Available from. URL: http://www.statistikbanken.dk/statbank5a/default.asp?w=1600.

3. Martin GS, Mannino DM, Moss M: The effect of age on the development and outcome of adult sepsis. Crit Care Med 2006, 34(1):15-21.

4. Wolff $\lrcorner$, Starfield B, Anderson G: Prevalence, expenditures, and complications of multiple chronic conditions in the elderly. Arch Intern Med 2002, 162(20):2269-2276.
5. Martin GS, Mannino DM, Eaton S, Moss M: The epidemiology of sepsis in the United States from 1979 through 2000. N Engl J Med 2003, 348(16):1546-1554.

6. Benfield $T$, Espersen F, Frimodt-Moller N, Jensen AG, Larsen AR, Pallesen LV et al: Increasing incidence but decreasing in-hospital mortality of adult Staphylococcus aureus bacteraemia between 1981 and 2000 Clin Microbiol Infect 2007, 13(3):257-263.

7. Sogaard M, Norgaard M, Dethlefsen C, Schonheyder HC: Temporal changes in the incidence and 30-day mortality associated with bacteremia in hospitalized patients from 1992 through 2006: a population-based cohort study. Clin Infect Dis 2011, 52(1):61-69.

8. Pedersen CB, Gotzsche H, Moller JO, Mortensen PB: The Danish Civil Registration System. A cohort of eight million persons. Dan Med Bull 2006, 53(4):441-449.

9. Jessen O, Rosendal K, Bülow P, Faber V, Eriksen KR: Changing staphylococci and staphylococcal infections. A ten-year study of bacteria and cases of bacteremia. N Engl J Med 1969, 281:627-635.

10. Skov RL, Pallesen LV, Poulsen RL, Espersen F: Evaluation of a new 3-h hybridization method for detecting the mecA gene in Staphylococcus aureus and comparison with existing genotypic and phenotypic susceptibility testing methods. J Antimicrob Chemother 1999, 43(4):467-475

11. Larsen AR, Stegger M, Bocher S, Sorum M, Monnet DL, Skov RL: Emergence and characterization of community-associated methicillin-resistant Staphyloccocus aureus infections in Denmark, 1999 to 2006. J Clin Microbiol 2009, 47(1):73-78.

12. Wacholder S, Silverman DT, McLaughlin JK, Mandel JS: Selection of controls in case-control studies. II. Types of controls. Am J Epidemiol 1992, 135(9):1029-1041.

13. Charlson ME, Pompei P, Ales KL, MacKenzie CR: A new method of classifying prognostic comorbidity in longitudinal studies: development and validation. J Chronic Dis 1987, 40(5):373-383.

14. Sundararajan V, Henderson T, Perry C, Muggivan A, Quan H, Ghali WA: New ICD-10 version of the Charlson comorbidity index predicted in-hospital mortality. J Clin Epidemiol 2004, 57(12):1288-1294.

15. De Groot V, Beckerman H, Lankhorst GJ, Bouter LM: How to measure comorbidity. a critical review of available methods. J Clin Epidemiol 2003, 56(3):221-229

16. Linderoth $G$, Jepsen P, Schonheyder HC, Johnsen SP, Sorensen HT: Shortterm prognosis of community-acquired bacteremia in patients with liver cirrhosis or alcoholism: A population-based cohort study. Alcohol Clin Exp Res 2006, 30(4):636-641.

17. Harboe ZB, Thomsen RW, Riis A, Valentiner-Branth P, Christensen JJ, Lambertsen $L$, et al: Pneumococcal serotypes and mortality following invasive pneumococcal disease: a population-based cohort study. PLoS Med 2009, 6(5):e1000081.

18. Laupland KB, Ross T, Gregson DB: Staphylococcus aureus bloodstream infections: risk factors, outcomes, and the influence of methicillin resistance in Calgary, Canada, 2000-2006. J Infect Dis 2008, 198(3):336-343.

19. Laupland KB, Church DL, Mucenski M, Sutherland LR, Davies HD: Population-based study of the epidemiology of and the risk factors for invasive Staphylococcus aureus infections. J Infect Dis 2003, 187(9):1452-1459.

20. Wisplinghoff $H$, Seifert $H$, Tallent SM, Bischoff $T$, Wenzel RP, Edmond MB Nosocomial bloodstream infections in pediatric patients in United States hospitals: epidemiology, clinical features and susceptibilities. Pediatr Infect Dis J 2003, 22(8):686-691.

21. Lyytikainen $\mathrm{O}$, Ruotsalainen $\mathrm{E}$, Jarvinen A, Valtonen $\mathrm{V}$, Ruutu P: Trends and outcome of nosocomial and community-acquired bloodstream infections due to Staphylococcus aureus in Finland, 1995-2001. Eur J Clin Microbiol Infect Dis 2005, 24(6):399-404.

doi:10.1186/1471-2334-12-260

Cite this article as: Mejer et al:: Stable incidence and continued improvement in short term mortality of Staphylococcus aureus bacteraemia between 1995 and 2008. BMC Infectious Diseases 2012 $12: 260$ 